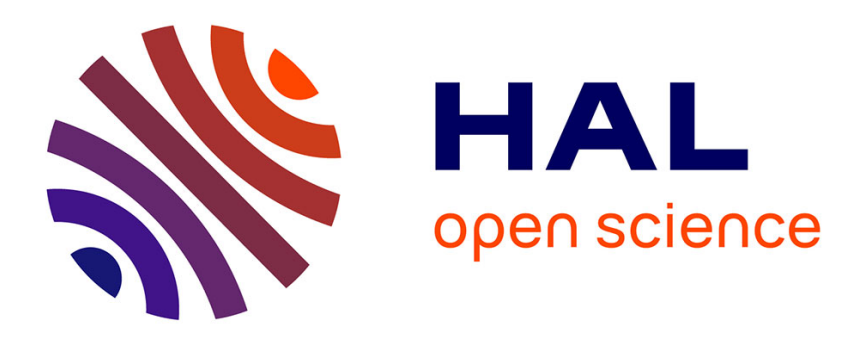

\title{
Shear-induced transition to mean-field critical behavior
}

\author{
D. Beysens, M. Gbadamassi
}

\section{- To cite this version:}

D. Beysens, M. Gbadamassi. Shear-induced transition to mean-field critical behavior. Journal de Physique Lettres, 1979, 40 (21), pp.565-567. 10.1051/jphyslet:019790040021056500 . jpa-00231689

\section{HAL Id: jpa-00231689 https://hal.science/jpa-00231689}

Submitted on 1 Jan 1979

HAL is a multi-disciplinary open access archive for the deposit and dissemination of scientific research documents, whether they are published or not. The documents may come from teaching and research institutions in France or abroad, or from public or private research centers.
L'archive ouverte pluridisciplinaire HAL, est destinée au dépôt et à la diffusion de documents scientifiques de niveau recherche, publiés ou non, émanant des établissements d'enseignement et de recherche français ou étrangers, des laboratoires publics ou privés. 


\title{
Shear-induced transition to mean-field critical behavior
}

\author{
D. Beysens and M. Gbadamassi \\ DPh-G/PSRM, CEN-Saclay, B.P. no 2, 91190 Gif-sur-Yvette, France
}

(Reçu le 2 juillet 1979, accepté le 20 septembre 1979)

\begin{abstract}
Résumé. - On utilise la diffusion de la lumière pour étudier l'influence d'un cisaillement $S$ sur les fluctuations critiques (temps de vie $\tau$ ) d'un mélange binaire (cyclohexane-aniline). Pour la $1^{\text {re }}$ fois nous montrons expérimentalement que dans la région $S \tau>1$ (i) la température critique est réellement changée $\Delta T_{\mathrm{c}}(S) \propto S^{0,53 \pm 0,03}$ et (ii) la susceptibilité mesurée dans la direction de la vitesse passe en comportement de champ moyen. Ces points confirment à la fois la réduction et l'anisotropie des fluctuations induites par le cisaillement.
\end{abstract}

\begin{abstract}
Light scattering was used to study the influence of a shear $S$ on the critical fluctuations (lifetime $\tau$ ) of a binary fluid (cyclohexane-aniline). For the first time we experimentally show that in the region $S \tau>1$ (i) the critical temperature is actually changed $\Delta T_{\mathrm{c}}(S) \propto S^{0.53 \pm 0.03}$ and (ii) the susceptibility measured in the flow direction crosses over to a mean-field behavior. These points confirm both the reduction and the anisotropy of the fluctuations induced by the shear.
\end{abstract}

In 1976, following a suggestion by P. Bergé, we started light scattering experiments concerning the influence of a shear flow on critical fluctuations. We have discovered [1] that when a binary mixture (cyclohexane-aniline) with concentration fluctuations of lifetime $\tau$ is submitted to a shear of rate $S\left(\mathrm{~s}^{-1}\right)$, three striking features appear :

(i) An effect is visible and detectable only in the region $S \tau>1$.

(ii) The turbidity decreases with increasing $S$ as if the critical temperature $T_{\mathrm{c}}$ were lowered, so that $\Delta T_{\mathrm{c}}(S)=T_{\mathrm{c}}(0)-T_{\mathrm{c}}(S) \propto S^{0.53}$.

(iii) A noticeable anisotropy with respect to the flow direction is seen in the scattered light, revealing a reduction of the fluctuations in the shear direction.

Subsequently Onuki and Kawasaki [2] (O. K.) have theoretically studied the same problem and reached the same general conclusions. In particular they have predicted that in the range $S \tau>1$, (i) a real change in $T_{\mathrm{c}}$ would occur,

$$
\Delta T_{\mathrm{c}}(S)=\frac{1}{12}\left[\frac{5 \pi \eta \xi_{0}^{3}}{k T}\right]^{1 / 3 v} \cdot S^{1 / 3 v}
$$

Here $v \simeq 0.63$ is the usual critical exponent of the correlation length in fluids, $\eta$ is the viscosity of the mixture $\left(\simeq 1.78 \times 10^{-2} P_{0}\right), \xi_{0}$ is the correlation length factor $\left(\simeq 2.45 \times 10^{-8} \mathrm{~cm}\right)$ and $T$ the absolute temperature $\left(\simeq T_{\mathrm{c}}=303 \mathrm{~K}\right)$, (ii) the temperature dependence of the scattered intensity is expected to follow an unusual behavior, crossing over from a normal behavior $\left[T-T_{\mathrm{c}}(0)\right]^{-\gamma}$, where $\gamma \simeq 1.24$, in the region $S \tau<1$, to a mean-field behavior $\left[T-T_{\mathrm{c}}(S)\right]^{-\gamma}$ with $\gamma^{\prime}=1$, in the region $S \tau>1$.

In this paper we report the first experimental observation of these two points. We performed new experiments with the same set-up as previously described in ref. [1] : The shear flow was produced in a rectangular quartz pipe and the scattering volume, illuminated by a He-Ne laser, is situated close to a side wall where the velocity gradient is nearly constant. The corresponding shear varies during the run and follows the law $S(t)=S_{\mathrm{M}} \cdot \exp \left(-t / \tau_{0}\right)$ where $\tau_{0}=13 \mathrm{~s}$ and $S_{\mathrm{M}}=1600 \mathrm{~s}^{-1}$. The extra-heating due to the shear flow was shown to be lower than $0.1 \mathrm{mK}$ and is thus negligibly small. The whole system is immersed in a water bath giving thermal stabilization $\pm 0.2 \mathrm{mK}$ over more than one hour, as verified by a quartz thermometer.

We have studied both the transmitted and the scattered light intensities. By improving the reproductibility of our measurements it was no longer necessary to calibrate the measurements performed during the run (at shear $S$ and temperature $T$ ) to the corresponding measurements performed at the end of the run when the flow is stopped (at shear $S=0$ and the same temperature $T$ ).

1. $\boldsymbol{T}_{\mathrm{c}}$ change. - By comparing the transmitted light intensities $\mathcal{C}$ with and without shear we have already deduced [1] an apparent $T_{\mathrm{c}}$ change. Indeed the simple change of $\left[T-T_{\mathrm{c}}(0)\right]$ into $\left[T-T_{\mathrm{c}}(S)\right]$ with 
$T_{\mathrm{c}}(S)<T_{\mathrm{c}}(0)$ has shown that the transmitted light intensities with shear $\mathcal{E}\left[T-T_{\mathrm{c}}(0), S\right]$ and without shear $\mathcal{C}\left[T-T_{\mathrm{c}}(S), 0\right]$ match well within experimental uncertainties. We have thus deduced

$$
\begin{aligned}
\Delta T_{\mathrm{c}}(S)=T_{\mathrm{c}}(0)-T_{\mathrm{c}}(S) \simeq(1.8 \pm 0.2) & 10^{-4} \times \\
& \times S^{0.53 \pm 0.03}
\end{aligned}
$$

which is in good agreement with the expected behavior calculated by O. K. :

$$
\Delta T_{\mathrm{c}}(S) \simeq 1.3 \times 10^{-4} \times S^{1 / 3 v} .
$$

In order to assess the physical reality of this $T_{\mathrm{c}}$ change, we performed new transmission recordings at temperatures $T$ below $T_{\mathrm{c}}(0)$. The mixture was stirred before the run in order to keep it homogeneous before the shear was applied. Five different types of variations, as shown in figure 1 , are evidenced :

(a) $T>T_{S}\left(S_{\mathrm{M}} \tau=1\right)$, where $\mathcal{C}$ is not effected by $S$.

(b) $T_{S}\left(S_{\mathrm{M}} \tau=1\right)>T>T_{\mathrm{c}}(0)$, where $\mathscr{C}$ decreases when $t$ increases and goes asymptotically to the equilibrium value.

(c) $T=T_{\mathrm{c}}(0)$, where $\mathscr{C}$ decreases and goes asymptotically to zero.

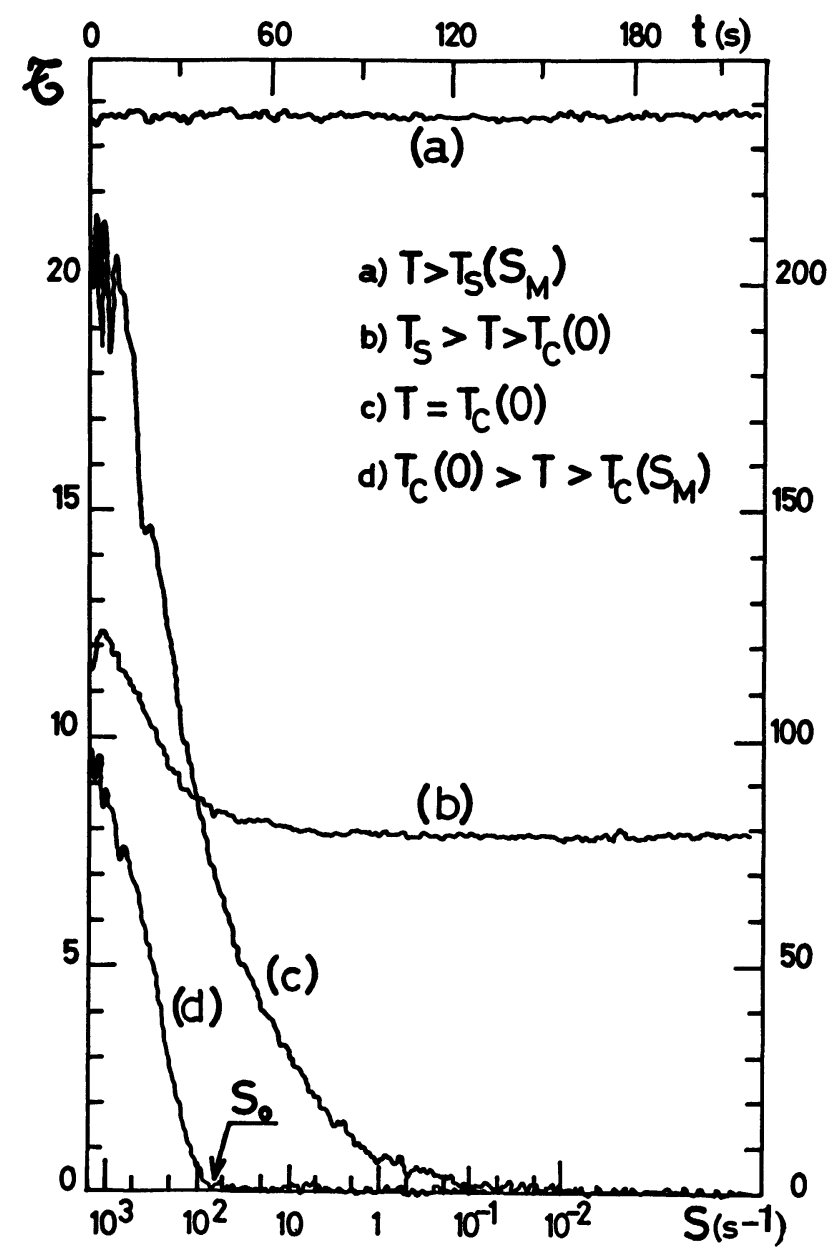

Fig. 1. - Behavior of the transmitted intensity $\prec$ versus shear $S$ or time $t \propto-\tau_{0} \mathrm{~L}_{\mathrm{N}}\left(S / S_{\mathrm{M}}\right)$ at different temperatures (see text). The right hand scale corresponds to $(a, b)$, and the left hand scale to $(c, d)$. (d) $T_{\mathrm{c}}(0)>T \geqslant T_{\mathrm{c}}\left(S_{\mathrm{M}}\right)$, where $\mathcal{C}$ falls abruptly to zero at the shear $S_{0}$.

(e) $T_{\mathrm{c}}\left(S_{\mathrm{M}}\right)>T$, where $\mathcal{C}$ remains equal to zero.

The value at which $\mathscr{C}=0$ corresponds exactly to the beginning of the phase separating process. Consequently, the behavior of $\mathcal{C}$ in $(d)$ shows unambiguously that the shear actually lowers $T_{\mathrm{c}}$. It is therefore possible to deduce the variation of $\Delta T_{\mathrm{c}}=T_{\mathrm{c}}(0)-T$ with shear $S_{0}$ and compare the present results with our previous data.

Figure 2 shows the close agreement between these two determinations of $\Delta T_{\mathrm{c}}(S)$. We checked moreover that the scattered light intensity exhibited no anomaly in the range $T_{\mathrm{c}}(0)>T>T_{\mathrm{c}}\left(S_{0}\right)$.

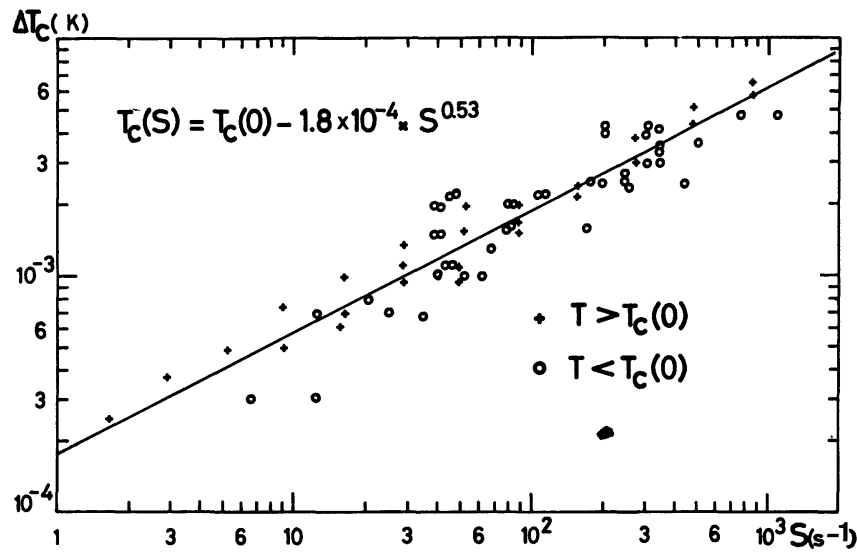

Fig. 2. - Critical temperature change versus shear $S$. + Already published data from the $\mathcal{C}$ variation above $T_{\mathrm{c}}(0)$. $\bigcirc$ New data from the determination of $S_{0}$ below $T_{\mathrm{c}}(0)$.

On the other hand, since the shear is seen to instantaneously change $T_{\mathrm{c}}$, this could be developed as a new method for studying phenomena such as spinodal decomposition and the expected change in the coexistence curve, etc...

2. Mean-field behavior. - According to O. K. [2], in the temperature and shear regions where $S \tau>1$, and for small transfer wavevector $\mathbf{k}$, the scattered intensity can be written as :

$I_{k}^{-1}=A S^{(2 v-1) / 3 v}\left[\frac{T-T_{\mathrm{c}}(S)}{T_{\mathrm{c}}}\right]^{\gamma^{\prime}}+B S^{8 / 15}\left|k_{x}\right|^{2 / 5}+k^{2}$

where $\gamma^{\prime}=1$.

$k_{x}$ is the projection of $\mathbf{k}$ on the direction $(X)$ of the flow.

$$
A=\left(\frac{5 \pi \eta}{k T}\right)^{(2 v-1) / 3 v} \xi_{0}^{-1 / v} \simeq 7.6 \times 10^{13} \text { c.g.s. }
$$

and

$$
B=\left(\frac{5 \pi \eta}{k T}\right)^{8 / 15} \simeq 7.0 \times 10^{6} \text { c.g.s. }
$$


We performed new experiments at small scattering angle $\left(\theta=2^{\circ}, k=5200 \mathrm{~cm}^{-1}\right)$. In the studied temperature, scattering angle, and shear domains, the $k^{2}$ term is negligible. The 2 nd term in (2) (the anisotropy term), is weak but not negligible. It would of course be better to perform experiments with $k_{x}=0$, i.e. perpendicular to the flow. In this case however the fluctuations of the scattered light are so intense and at so low a frequency that the accuracy of the data is not very high, which contrasts with the $X$ direction where a Doppler effect takes place. We have thus performed the experiments only in the flow direction. Figure 3 represents a $\log -\log$ plot of $I$ versus $T-T_{\mathrm{c}}(S)$ for different values of $S$. It is clear that the cross-over temperature $T_{S}(S)$ (where $S \tau=1$ ) corresponds to a change of behavior. In figure 3 is also plotted the best fit of experimental results with $S=0$, i.e. the usual variation for a fluid at equilibrium, $I_{k}^{-1}=\left(k^{2}+\xi^{-2}\right)^{1-\eta / 2}$ where $\eta \simeq 0.031$ is the Fisher exponent and $\xi=\xi_{0}\left(\frac{T-T_{\mathrm{c}}}{T_{\mathrm{c}}}\right)^{-v}$ is the correlation length. We have not been able to fit our data with the O. K. formula, due perhaps to the anisotropy term in (2) which seems to be lower than expected. This point needs further investigation. But it is clear that the temperature variation can be well described by the mean-field dependence, excepted for the highest shear values where a rounding-off begins to be visible (anisotropy term).

This is the first time to our knowledge that such a change in the behavior is seen in a critical system of dimensionality $d=3$ and with a scalar $(n=1)$ order parameter. It is well known [3] that the mean-field behavior is relevant when the fluctuations of the order parameter $(c)$ remain negligible with respect to its mean value. For small values of the wavevector $k$, and by simply assuming that the anisotropy due to the shear can be written as a series expansion in $k_{x}^{p} S^{m}$, the mean-field behavior would be evidenced if the conuribution from the fluctuations is negligible, even at $T_{\mathrm{c}}$, i.e. if $\int_{0}^{1 / \xi_{0}} \frac{\mathrm{d}^{3} k}{k_{x}^{2 p}}$ remains finite, which is verified for $p<3 / 2$. This condition is actually fulfilled as can be seen in ref. [1] when plotting from figure 2 $\ell_{\mathrm{N}} I$ versus $l_{\mathrm{N}} k_{x}$, leading to the conclusion that it is merely the reduction of the amplitude of the order parameter fluctuations which involves the mean-field behavior.

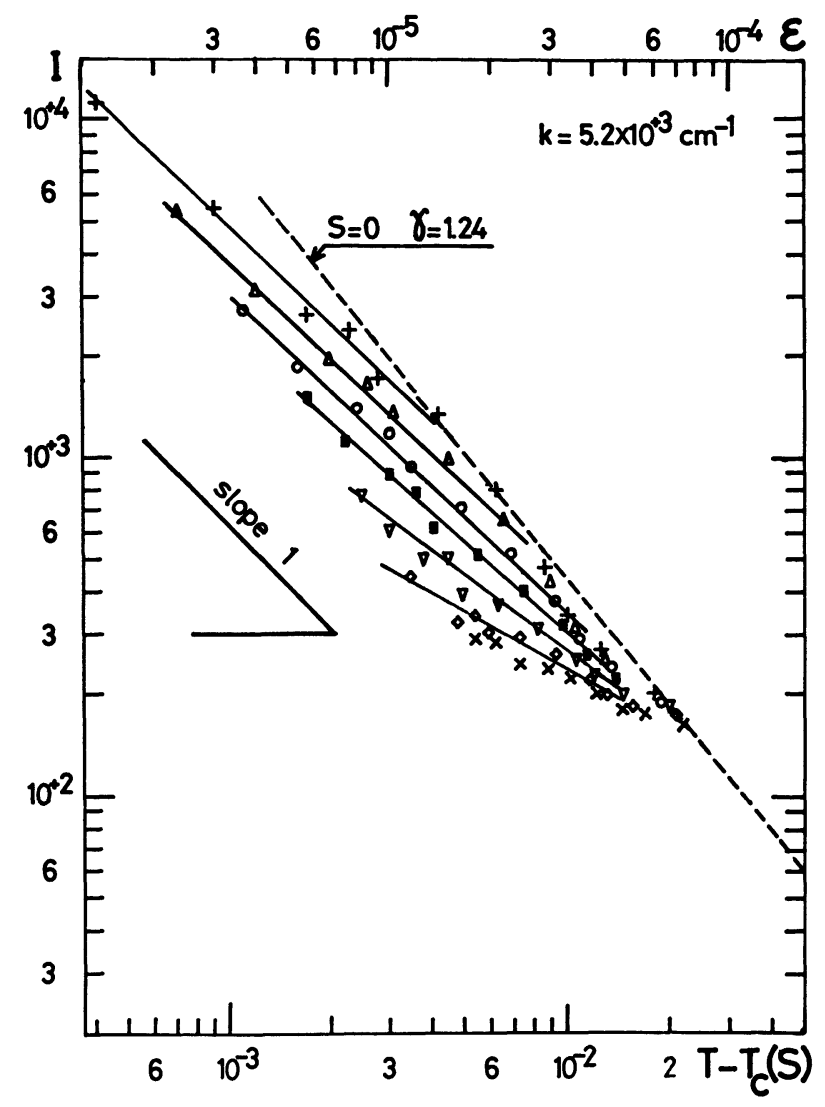

Fig. 3. - Scattered intensity $I$ versus $T-T_{\mathrm{c}}(S)$ at various shears $S: \times 903 \mathrm{~s}^{-1} ; \diamond 510 \mathrm{~s}^{-1} ; \nabla 288 \mathrm{~s}^{-1} ; 163 \mathrm{~s}^{-1} ; 092 \mathrm{~s}^{-1}$; $\triangle 52 \mathrm{~s}^{-1} ;+29 \mathrm{~s}^{-1}$. The dotted line is the best fit to all experimental data obtained with $S=0$, and corresponds to the normal behavior $\simeq\left(T-T_{\mathrm{c}}\right)^{-1.24}$.

Conclusion. - When a shear flow is applied to a critical binary fluid $(n=1, d=3)$, we have experimentally shown that the critical behavior is completely changed : the temperature of the phase separation process is lowered by an amount $\Delta T_{\mathrm{c}}(S) \propto S^{0.53}$. The shear reduces the amplitude of the order parameter fluctuations while introducing an anisotropy. The mean-field behavior thus becomes relevant as shown by the susceptibility which is seen, in the region $S \tau=1$, to cross over from an usual temperature dependence in fluids $(S \tau<1)$ with the exponent $\gamma=1.24$, to a mean-field behavior $(S \tau>1)$ with the exponent $\gamma^{\prime}=1$.

Acknowledgments. - We are particularly indebted to P. Bergé and E. Brezin for fruitful discussions.

\section{References}

[1] Beysens, D., Gbadamassi, M. and Boyer, L., to appear in Phys. Rev. Lett.

[2] Onuki, A. and KawaSaki, K., to be published in Ann. Phys. (N-Y); Progr. Theor. Phys. Supplement; Phys. Lett. A.

[3] See for instance Boccara, N., Symétries Brisées (Hermann, Paris) 1976. 